\title{
Cyanidin ameliorates cisplatin-induced cardiotoxicity via inhibition of ROS-mediated apoptosis
}

\author{
PENG QIAN, LI-JIE YAN, YONG-QIANG LI, HAI-TAO YANG, \\ HONG-YAN DUAN, JIN-TAO WU, XIAN-WEI FAN and SHAN-LING WANG
}

Department of Cardiology, Henan Provincial People's Hospital, Zhengzhou, Henan 450003, P.R. China

Received February 23, 2017; Accepted August 17, 2017

DOI: $10.3892 /$ etm.2017.5617

\begin{abstract}
Oxidative stress and apoptosis serve an essential role in cisplatin-induced cardiotoxicity, which limits its clinical use, and increases the risk of cardiovascular disease. As a natural drug, the antioxidant and antitumor effects of cyanidin have been recognized, but its protective effect on cisplatin-induced cardiomyocyte cytotoxicity remains unclear. H9c2 cells were treated with cisplatin $(1-40 \mu \mathrm{M})$ in the presence or absence of cyanidin (40-80 $\mu \mathrm{M})$, subsequently; oxidative stress, apoptosis and mitochondrial function were assessed using several techniques. The results demonstrated that cyanidin was able to dose-dependently reverse cisplatin-induced cell damage and apoptosis, attenuate the accumulation of reactive oxygen species (ROS), and mitochondrial membrane potential depolarization, downregulate the expression of $\mathrm{Bcl}-2$ homologous antagonist/killer, upregulate the expression of apoptosis regulator Bcl-2, and reduce the activation of caspase 3, caspase 9 , but not caspase 8 . Furthermore, the results revealed that the translocation of apoptosis regulator Bax (Bax) from the cytoplasm to the mitochondrial membrane serves an essential role in cisplatin-induced apoptosis. Cyanidin was able to block the translocation of Bax and reduce the release of cytochrome $c$ from cytoplasm. These data indicate that cyanidin attenuates cisplatin-induced cardiotoxicity by inhibiting ROS-mediated apoptosis, while the mitochondrial and extracellular regulated kinase signaling pathways may also serve important roles.
\end{abstract}

Correspondence to: Dr Shan-Ling Wang, Department of Cardiology, Henan Provincial People's Hospital, 7 Wei Five Road, Jinshui, Zhengzhou, Henan 450003, P.R. China

E-mail: wangshanling2002@126.com

Abbreviations: ROS, reactive oxygen species; MMP, mitochondrial membrane potential; ERK, extracellular signal-regulated kinase; JNK, c-JUN N-terminal kinase; MAPK, Mitogen activated protein kinases

Key words: cardiotoxicity, cisplatin, cyanidin, oxidative stress, apoptosis, ERK pathway

\section{Introduction}

Cisplatin, a commonly used cytotoxic antitumor drugs, has a major clinical efficacy, but dose related cardiotoxicity are increasingly recognized among clinicians (1), which contains electrocardiographic changes, arrhythmias $(2,3)$, angina pectoris (4), cardiac failure (5), vascular events (6) etc. These events may restrict dose of cisplatin in some cases and increase the long-term prevalence of cardiovascular disease in patients have been received chemotherapy. The current study suggests that cisplatin injury mainly in myocardial cells and vascular endothelium, eventually leads to myocardial cell systolic dysfunction associated with mitochondrial damage and vascular endothelial injury (7). Despite the precise mechanisms of cisplatin-induced cardiotoxicity is not fully elucidated, oxidative stress, apoptosis, DNA damage, endoplasmic reticulum and inflammation are considered to be involved in the occurrence of cells injury (1).

Cisplatin can break the balance of intracellular oxidative stress and antioxidant stress when it entered the cell, which leading to increased oxygen free radicals and causing two different outcomes, apoptosis and necrosis (8), and the former has been confirmed in a variety of cytotoxicity models induced by cisplatin (9-11). Bcl-2 family plays an important role in the process of cardiomyocytes apoptosis, and Bax and Bcl-2 are the most representative inhibition of apoptosis and promote apoptosis gene, and Bax is the main regulator of $\mathrm{Bcl}-2$ activity. When cisplatin induces generation of ROS, Bax was activated and transported to the mitochondrial outer membrane and changes its permeability, causing the opening of the mitochondrial permeability transition pores (MPTPs), releasing the cytochrome $c$ into the cytosol, and leading to activation of caspase 9, as well as downstream caspases and inducing caspase-dependent manner (10).

Mitogen activated protein kinases (MAPK) are a family of structurally related serine/threonine protein kinase that transduces extracellular signals into the cells to regulate cell growth, proliferation and differentiation, including c-JUN N-terminal kinase (JNK), extracellular signal-regulated kinase (ERK) and p38 kinase. Cisplatin has been shown to modulate the ERK signal pathway to induce apoptosis in a variety of cells, but its mechanism of action on cardiomyocytes remains unclear (11).

As a member of the flavonoid family, anthocyanins have received more and more attention in clinical application due 
to their clear antioxidant action. Among of them, cyanidin has been extensively studied because of their novel anticancer and anti-apoptosis activities $(12,13)$. Shih et al found that cyanidin could play antioxidant protection capacity through the Nrf2/ARE pathway (14). Park et al demonstrated that cyanidin had a strong inhibitory effect on tumorigenic transformation via different cellular signaling pathways (15). Moreover, cyanidin attenuated ROS-mediated apoptosis have been demonstrated in HK2 cells and PC12 cells $(16,17)$. Increasing studies have focused on the use of natural drugs in combination with chemotherapy to relieve the side effects of the latter, but little is known about the mechanism of cisplatin induced cardiotoxicity. Therefore, in the present study, H9c2 cardiomyocytes were used as a model to investigate the mechanism of cyanidin against cisplatin-induced H9c2 cardiomyocytes damage.

\section{Materials and methods}

Cell culture. An H9c2 cardiac myoblast cell line was obtained from the Shanghai Institute of Biochemistry and Cell Biology, Shanghai Institutes for Biological Sciences, the Chinese Academy of Sciences (Shanghai, China). The cells were cultured in DMEM medium (containing 10\% fetal bovine serum, $1 \%$ penicillin/streptomycin, and $25 \mathrm{mM}$ Hepes) at $37^{\circ} \mathrm{C}$ in a humidified incubator with $5 \% \mathrm{CO}_{2}$ for $48 \mathrm{~h}$. Cisplatin was dissolved in water and diluted to $0,5,10,20,40 \mu \mathrm{M}$, respectively. Cyanidin were prepared in dimethyl sulfoxide (DMSO) and diluted to $0,40,80 \mu \mathrm{M}$. The final concentration of DMSO was kept under $1 \%$. Control wells contained cells without drug and, in some cases with $0.2 \%$ of DMSO (v/v). For drug treatments, cells were seeded at $3 \times 10^{4}$ cells/well in 96-well plate and treated with $0-40 \mu \mathrm{M}$ of cisplatin for $24 \mathrm{~h} 40-80 \mu \mathrm{M}$ cyanidin for $24 \mathrm{~h}$. Pre-treatment of cyanidin was carried out $3 \mathrm{~h}$ prior to the application of cisplatin.

MTT assay. Cell viability was detected by MTT assay. $\mathrm{H} 9 \mathrm{c} 2$ cells plated in 96 -well plates at $3 \times 10^{4}$ cells/well were pre-treated with cyanidin for $3 \mathrm{~h}$ and co-treated with cisplatin for $24 \mathrm{~h}$, and then cells were incubated with different treatments and different times. After incubation, the medium was removed and $10 \mu \mathrm{l}$ MTT $(5 \mathrm{mg} / \mathrm{ml})$ (Sigma-Aldrich, Saint Louis, Missouri, USA) was added for $4 \mathrm{~h}$. After the incubation, $150 \mu 1$ DMSO was added to dissolve formazan crystals, and the absorbance was measured at $570 \mathrm{~nm}$ with a microplate reader Elx800 (Bio-Tek, Winooski, Vermont, USA).

TUNEL assay. Treated H9c2 cells cultured in 6-well plates were fixed with $4 \%$ neutral formaldehyde for $20 \mathrm{~min}$ at $4^{\circ} \mathrm{C}$. After washing with PBS, cells were permeabilized on ice with $0.1 \%$ Triton X-100 in PBS for 2 min. Washed cells were incubated for $60 \mathrm{~min}$ with $50 \mu \mathrm{l} /$ well TUNEL reaction mixture (Beyotime, Shanghai, China), which containing nucleotide mixture and terminal deoxynucleotidyl transferase. At last, the cells were then washed by PBS for 3 times and observed under fluorescence microscope (Olympus BX43, Tokyo, Japan). TUNEL-positive cells were random counted in 5 randomly fields per section, then quantified by image analysis system. The apoptosis rate was expressed as the ratio of TUNEL-positive cardiomyocytes number to total number of cardiomyocytes.
Flow cytometry assay. The number of apoptotic cells was quantified using Annexin V-binding and PI-uptake (Sigma-Aldrich, St. Louis, MO, USA). H9c2 cells after treatment were collected by centrifugation, washed by PBS. $5 \mu \mathrm{l}$ Annexin V-FIT $(20 \mu \mathrm{g} / \mathrm{ml})$ and $10 \mu \mathrm{l} \mathrm{PI}(50 \mu \mathrm{g} / \mathrm{ml})$ were added and incubated in dark at room temperature for $15 \mathrm{~min}$, then quantified by cell flow cytometry (BD, CA, USA).

Determination of intracellular ROS. The intracellular ROS was performed using a fluorescent probe (Beyotime, Shanghai, China). H9c2 Cells were seeded in 96-well microplates $\left(10^{5}\right.$ cells/well) were incubated with $10 \mu \mathrm{M}$ DCFH-DA at $37^{\circ} \mathrm{C}$ for $20 \mathrm{~min}$, washed by serum-free cell culture medium. Then cells were treated with cisplatin $1 \mathrm{~h}$ and or cyanidin $3 \mathrm{~h}$. After that, the ROS level was detected by Laser scanning confocal microscope (TCS SP5, Leica, Germany).

Mitochondrial membrane potential assay. H9c2 cells in 6-well plates were trypsinized after treated with cisplatin $1 \mathrm{~h}$ or/and cyanidin $3 \mathrm{~h}$. Then cells washed with PBS and resuspended in $0.5 \mathrm{ml}$ of PBS buffer containing $10 \mu \mathrm{g} / \mathrm{ml}$ of JC-1. After incubation for $30 \mathrm{~min}$ at $37^{\circ} \mathrm{C}$, cells were centrifuged to discard the supernatant. Cell pellets were suspended in PBS and then analyzed by flow cytometry. Mitochondrial membrane potential was measured using JC-1 Assay kit (Beyotime, Shanghai, China) according to the manufacturer's protocol. JC-1 is a mitochondria-sensitive dye that detects mitochondrial membrane potential changes by observing the degree of mitochondrial matrix aggregation. The aggregate JC-1 (red fluorescence) was detected at the emission wavelength of $590 \mathrm{~nm}$, and the monomeric JC-1 (green fluorescence) monitored at $529 \mathrm{~nm}$.

Caspase activity assay. Caspase 3, caspase 9 and caspase 8 activities were determined by spectrophotometric method (Caspase Assay kit, Beyotime, Jiangsu, China). Treated cells were harvested, suspended in cell lysis buffer (80-100 $\mu \mathrm{l})$ and incubated on ice for $30 \mathrm{~min}$, after centrifugation at $10,000 \mathrm{x} \mathrm{g}$ for $15 \mathrm{~min}$ at $4^{\circ} \mathrm{C}$, supernatants were collected and placed in 96-well plates and then $10 \mu \mathrm{l}$ specific caspase substrates (Ac-DEVD- $p$ NA) was added. Plates were incubated at $37^{\circ} \mathrm{C}$ for $2 \mathrm{~h}$ and caspase activities were tested by spectrophotometer (Spectrum, Shanghai, China).

Fractionation of cytoplasm and mitochondria protein. Cells were collected and washed by ice-cold PBS, and resuspended in buffer (250 mM sucrose, $1 \mathrm{mM}$ EDTA, $50 \mathrm{mMTris}-\mathrm{HCl}$, $1 \mathrm{mM}$ DTT, $1 \mathrm{mM}$ PMSF, $1 \mathrm{mM}$ Benzamidine, $0.28 \mathrm{u} / \mathrm{ml}$ apotinin, $50 \mu \mathrm{g} / \mathrm{ml}$ leupeptin, and $7 \mu \mathrm{g} / \mathrm{ml}$ pepstain A, $\mathrm{pH} 7.4$ ), then transferred to a glass homogenizer (Shanghai, China) and twitched 30 times. The lysates were centrifuged at $1,000 \mathrm{x} \mathrm{g}$ for $10 \mathrm{~min}$ at $4^{\circ} \mathrm{C}$, the supernatant was transferred to a new $\mathrm{EP}$ tube and subsequently centrifuged at 1,000 x $\mathrm{g}$ for $20 \mathrm{~min}$ at $4^{\circ} \mathrm{C}$, the supernatant contained cytoplasmic and membrane protein and the sediments was the crude of mitochondria. The cytosolicfractions was purified by ultracentrifugation and the crude of mitochondria was resuspended and centrifuged 3 times at $10,000 \mathrm{x}$ g for $10 \mathrm{~min}$ at $4^{\circ} \mathrm{C}$. Finally, mitochondria were precipitated in cell lysates (18). 

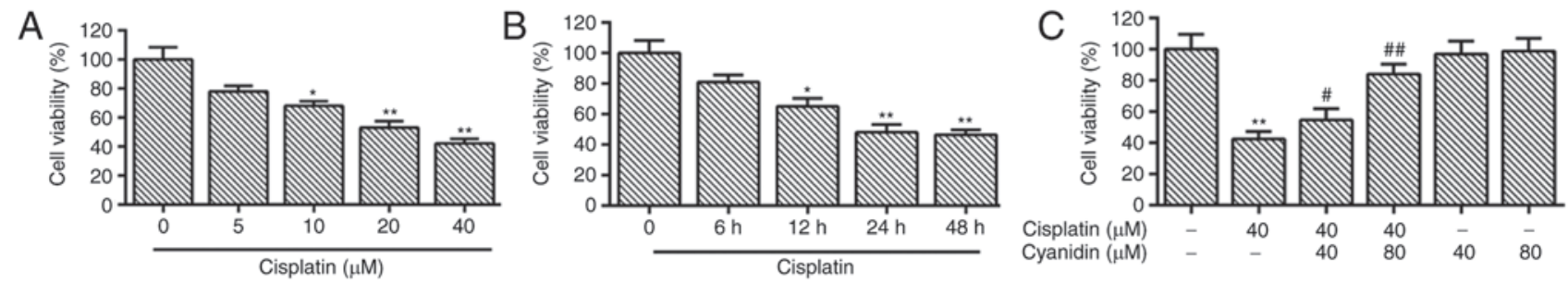

Figure 1. Effect of cisplatin on H9c2 cells viability and protective effects of cyanidin. (A) H9c2 cells were incubated with different concentrations of cisplatin $(0-40 \mu \mathrm{M})$ for $24 \mathrm{~h}$, cells viability were detected by MTT assay. (B) H9c2 cells were incubated with cisplatin for $0,6,24$ and $48 \mathrm{~h}$, cells viability were detected by MTT assay. (C) Cells were pre-treated with cyanidin ( $40-80 \mu \mathrm{M})$ for $3 \mathrm{~h}$, then exposed to cisplatin $(40 \mu \mathrm{M})$ for $24 \mathrm{~h}$. Data represent means $\pm \mathrm{SD}$, "P<0.05, ${ }^{* *} \mathrm{P}<0.01$ compared to control group, ${ }^{\#} \mathrm{P}<0.05,{ }^{* \#} \mathrm{P}<0.01$ compared with cisplatin group.
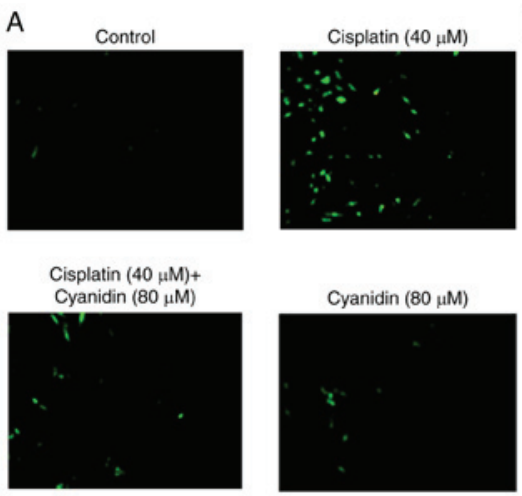

Cyanidin $(80 \mu \mathrm{M})$

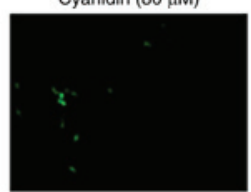

C

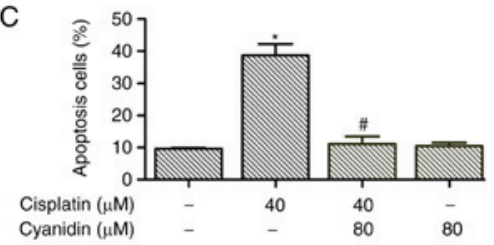

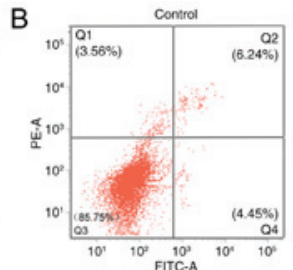
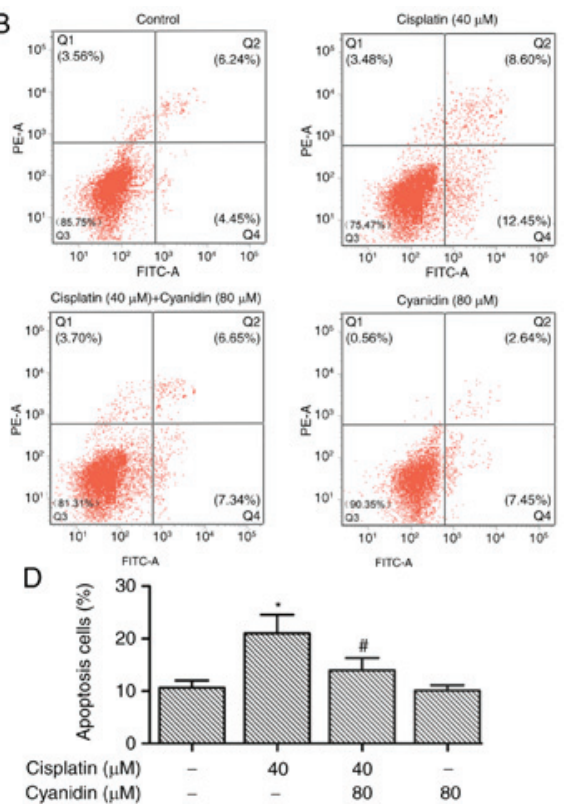

Figure 2. Cyanidin reduces cisplatin-induced H9c2 cell apoptosis. (A) Apoptotic cells were detected by TUNEL after incubated with cisplatin (40 $\mu \mathrm{M})$ alone or co-treated with cyanidin $(80 \mu \mathrm{M})$ for $24 \mathrm{~h}$. (B) Representation images of the effects of cyanidin pretreatment on cisplatin-induced apoptotic cell distribution in H9c2 cells. (C) The apoptosis rate was expressed as the ratio of TUNEL-positive cardiomyocytes number to total number of cardiomyocytes. (D) The statistical analysis of apoptotic cells. The apoptotic cells show hypodiploid DNA contents (Q3 area), the data were expressed in the histogram and analyzed by Modfit Software "P $<0.05$ compared with control group, ${ }^{\#} \mathrm{P}<0.05$ compared with cisplatin group.

Western blotting. Equal amounts of protein from each sample were resolved on SDS-PAGE (BioRad, USA) and transferred to polyvinylidene fluoride membranes (PVDF, Thermo Scientific, Rockford IL, USA). The membranes were blocked in TBS buffer containing 5\% nonfat milk for $1 \mathrm{~h}$, then incubated with primary antibodies at 1:1,000 dilutions overnight at $4{ }^{\circ} \mathrm{C}$ in $5 \%$ non-fat milk. Following, it was incubated with secondary antibodies at 1:2,000 for $1 \mathrm{~h}$ at room temperature. After washed by TBS buffer, immunoreactive bands on the membrane were visualized using chemiluminescent reagents (Thermo Scientific, Rockford IL, USA), the results analyzed with software (Tanon, Shanghai, China). The primary antibodies included cleaved caspase 3, Bax, Bak, Bcl-2, cytochrome $c$, ERK, p-ERK, $\beta$-actin, and Cox IV, all of which were purchased from Cell Signaling Technology, Inc.

Statistical analysis. Every experiment was repeated at least three times. Data were expressed in the form of mean \pm standard deviation. Student $t$ test was used to compare the differences between the two means and one-way ANOVA was used to compare the differences between multiple parameters. $\mathrm{P}<0.05$ was considered statistically significant.

\section{Results}

Cyanidin improves myocardial cells viability after exposure to cisplatin. To investigate the cytotoxic effects of cisplatin on $\mathrm{H} 9 \mathrm{c} 2$ cells and possible protective effects of cyanidin, the pre-treated cells were detected by MTT assay. As shown in Fig. 1, cultured H9c2 cells were incubated with varying concentrations of cisplatin $(0-40 \mu \mathrm{M})$ and periods of time, the results showed that the cell viability decreased with increasing cisplatin concentration (Fig. 1A). Similarly, with the prolongation of incubation time, the cell viability was significantly reduced (Fig. 1B), which implying potential cardiotoxicity. For combined intervention, cyanidin significantly reversed cisplatin-induced the decline of cells viability in a dose-dependent way (Fig. 1C). 
A
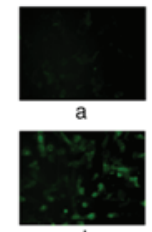

d
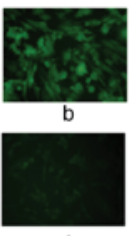
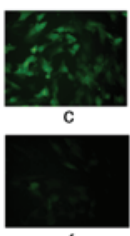

B

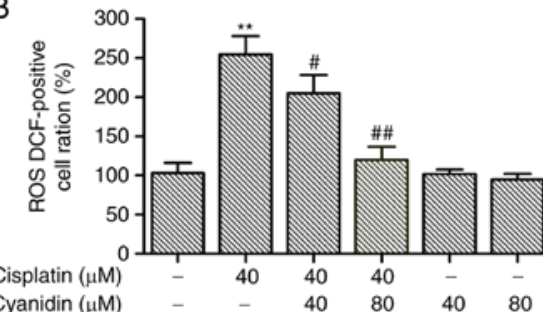

C
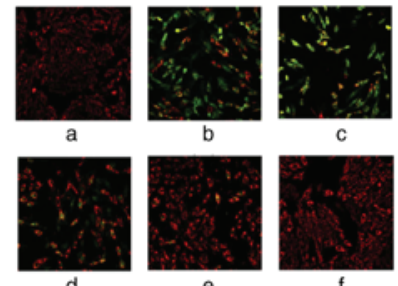

D

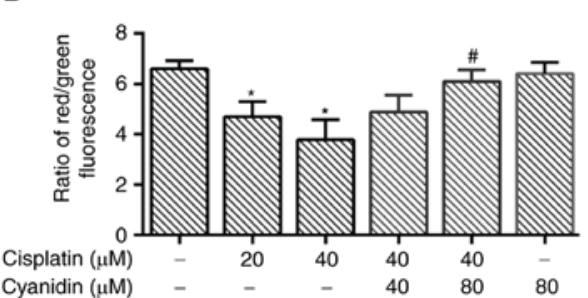

Figure 3. Cyanidin attenuates cisplatin-induced accumulation of ROS and $\Delta \Psi \mathrm{m}$ depolarization in H9c2 cells. (A) Representative images of ROS assay. (B) Quantitative analysis of ROS assay. (C) Representative images of JC-1 staining. (D) Quantitative analysis of JC-1 staining. (a) control; (b) cisplatin (40 $\mu \mathrm{M}$ ); (c) cisplatin $(40 \mu \mathrm{M})+$ cyanidin $(40 \mu \mathrm{M})$; (d) cisplatin $(40 \mu \mathrm{M})+$ cyanidin $(80 \mu \mathrm{M})$; (e) cyanidin $(40 \mu \mathrm{M})$; (f) cyanidin $(80 \mu \mathrm{M})$. Data represent means \pm SD, ${ }^{*} \mathrm{P}<0.05,{ }^{* *} \mathrm{P}<0.01$ compared to control group, ${ }^{\#} \mathrm{P}<0.05,{ }^{\# \#} \mathrm{P}<0.01$ compared with cisplatin $(40 \mu \mathrm{M})$ group.
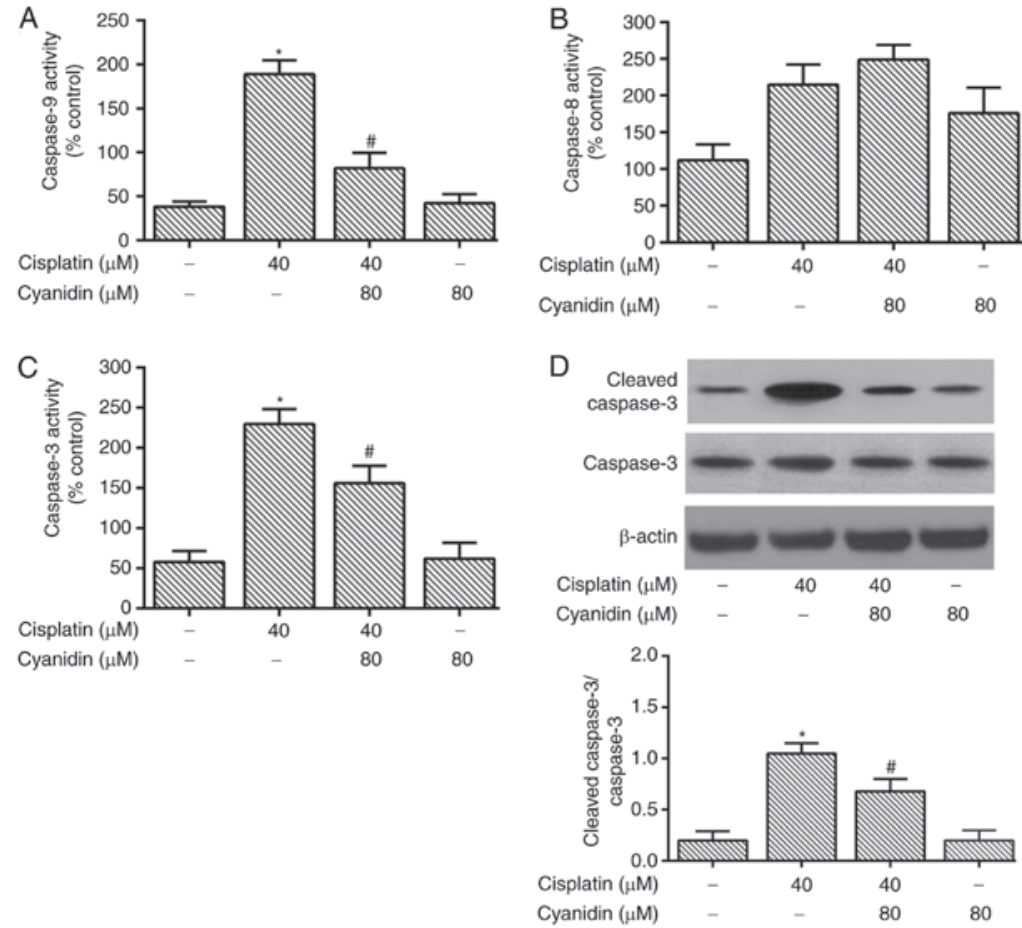

Figure 4. Cyanidin alleviates cisplatin-induced activation of caspases in H9c2 cells. (A-C) Caspase 9, caspase 8, and caspase 3 were detected by spectrophotometry. (D) After cells pretreatment, cleaved caspase 3 and caspase 3 proteins were extracted and detected by western blotting. All experiments were repeated twice with similar results and the data is normalized after grayscale scanning. Data represent means $\pm \mathrm{SD},{ }^{*} \mathrm{P}<0.05$ compared to control group, ${ }^{\#} \mathrm{P}<0.05$, compared with cisplatin group.

Cyanidin reduces cisplatin-induced $H 9 c 2$ cell apoptosis TUNEl and Annexin V-FITC/PI staining was used to detect apoptosis (Fig. 2). As shown in Fig. 2A and C, H9c2 cells apoptosis were detected by TUNEL after exposed with cisplatin alone or co-treated with cyanidin. The data indicated that cisplatin induced evident apoptosis compared with control group. However, co-treatment with cyanidin greatly reduced cells apoptosis. For more accurate results, Annexin V-FITC/PI was used to detect early stage of apoptosis. The results showed that $80 \mu \mathrm{h}$ cyanidin reduced the cisplatin-induced apoptotic rate (Fig. 2B and D), which were further confirmed that cyanidin inhibits cisplatin-induced apoptosis.

Cyanidin attenuates cisplatin-induced accumulation of ROS and $\Delta \Psi m$ depolarization. Oxidative stress-mediated cells damage is an important mechanism of cisplatin induced cytotoxicity. The present data show that the intracellular ROS increased significantly when exposed to cisplatin. After the combination with cyanidin 40 or $80 \mu \mathrm{M}$, the levels of ROS were markedly reduced (Fig. 3A and B). Overexpression of ROS can cause mitochondrial dysfunction, and then activate 

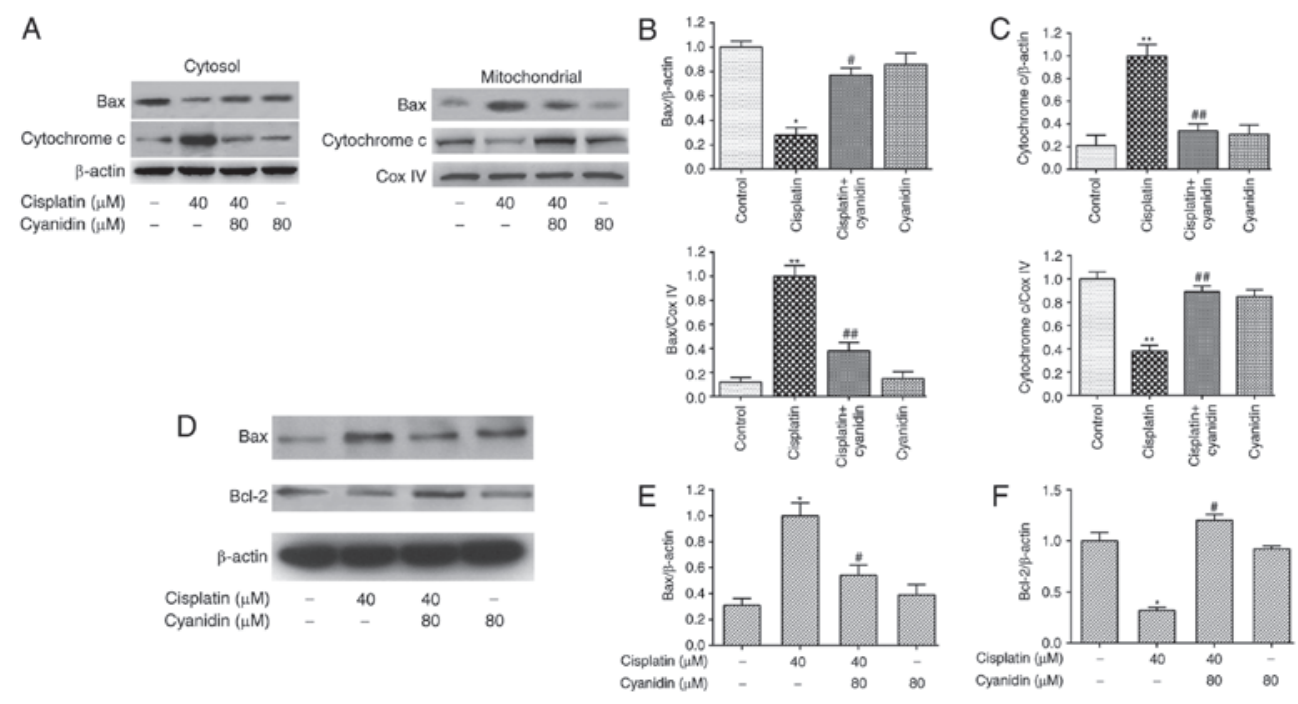

Figure 5. Cyanidin promotes the translocation of Bax and the expression of anti-apoptotic protein in H9c2 cells. (A) H9c2 cells were pre-treated with cisplatin $(40 \mu \mathrm{M})$ for $24 \mathrm{~h}$ in the presence or absence of cyanidin $(80 \mu \mathrm{M})$, then Bax and cytochrome $c$ located in the cytoplasm and mitochondria were extracted for immunblotting. (B) Relative quantification of Bax in cytoplasm and mitochondrial. (C) Relative quantification of cytochrome $c$ in cytoplasm and mitochondrial. (D) H9c2 cells were pre-treated same as (A and B) and the quantitative expression of Bak and Bcl-2 were detected by western blotting. (E) The ratio of Bak/ $\beta$-actin. (F) The ratio of Bcl-2/ $\beta$-actin. Data represent means $\pm \mathrm{SD},{ }^{*} \mathrm{P}<0.05,{ }^{* *} \mathrm{P}<0.01$ compared with control group, ${ }^{\#} \mathrm{P}<0.05,{ }^{\# \#} \mathrm{P}<0.01$ compared with cisplatin group.
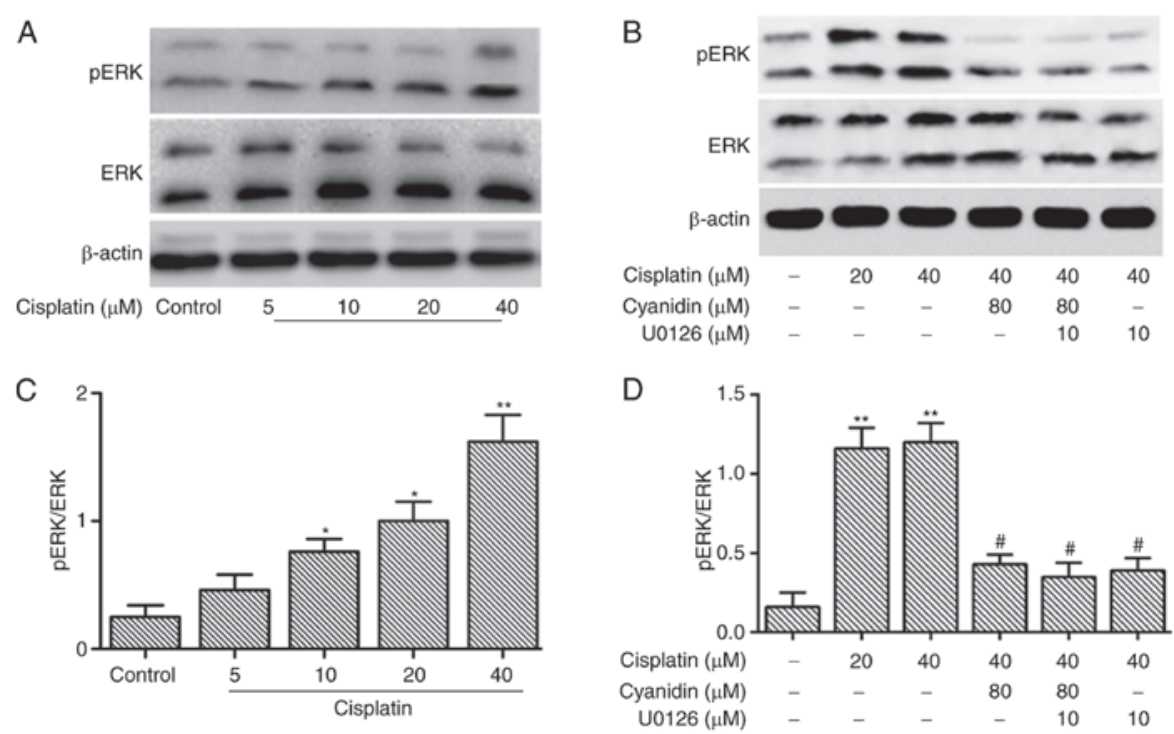

Figure 6. Cyanidin suppresses cisplatin-induced ERK activation. (A) H9c2 cells were pre-treated with cisplatin at varying doses for $24 \mathrm{~h}$, and immunblotting was performed to detected ERK and ERK phosphorylation. (B) H9c2 cells were incubated with cisplatin $(20 \mu \mathrm{M}, 40 \mu \mathrm{M})$ for $24 \mathrm{~h}$ in the presence or absence of cyanidin $(80 \mu \mathrm{M})$ and $\mathrm{U} 0126(10 \mu \mathrm{M})$, then tested by western blotting. (C) The ratio of pERK to ERK was expressed as a histogram. (D) The ratio of pERK/ERK in different groups. Data represent means $\pm \mathrm{SD},{ }^{*} \mathrm{P}<0.05,{ }^{* *} \mathrm{P}<0.01$ compared with control group, ${ }^{*} \mathrm{P}<0.05$ compared with cisplatin $(40 \mu \mathrm{M})$ group.

cell apoptosis. To verify this conclusion, H9c2 cells were incubated with cisplatin $(0,20,40 \mu \mathrm{M})$ for $24 \mathrm{~h}, 20$ and $40 \mu \mathrm{M}$ cisplatin all induced obviously decreased of $\Delta \Psi_{\mathrm{m}}$, and 40 and $80 \mu \mathrm{M}$ cyanidin all relieved cisplatin-induced loss of $\Delta \Psi \mathrm{m}$ (Fig. 3C), especially $80 \mu \mathrm{M}$ cyanidin significantly alleviated the elevated $\Delta \Psi \mathrm{m}$ (Fig. 3D).

Cyanidin alleviates cisplatin-induced activation of caspases. Several studies have demonstrated that cisplatin-induced cells toxicity is associated with both intrinsic and extrinsic apoptotic pathways $(10,19)$, to identify the exact pathway of cisplatin action, we test activity of caspase 9 , caspase 8 and caspase 3 by spectrophotometric method. As shown in Fig. 4, after cisplatin administration $(40 \mu \mathrm{M})$, the activity of caspase 9 , caspase 8 and caspase 3 were significantly increased. When co-incubated with cyanidin $(80 \mu \mathrm{M})$, the expression of caspase 9 and caspase 3 were evidently reduced (Fig. 4A and C), but not caspase 8 (Fig. 4B). Meanwhile, activation of caspase 3 was also detected by western blotting (Fig. 4D), it could also suppressed by cyanidin ( $80 \mu \mathrm{M})$. These results suggested that cyanidin mitigated cardiomyocytes apoptosis via mitochondrial pathway.

Cyanidin promotes the translocation of Bax and the expression of anti-apoptotic protein. As shown in Fig. 5, 
apoptosis-related proteins were detected by immunblotting. In the control group, Bax was mainly expressed in cytoplasm, and cytochrome $c$ was mainly expressed in mitochondria. After cisplatin administration, Bax was significantly decreased in cytoplasm and increased in mitochondria, whereas cytochrome $c$ showed an opposite tendency to Bax, which further leading to caspases activation. However, combined with cyanidin treatment reversed the translocation of Bax and cytochrome $c$ (Fig. 5A). The relative quantitative results also suggested that cyanidin treatment significantly improved the translocation of Bax induced by cisplatin (Fig. 5B and C). Moreover, we detected Bak and Bcl-2 by immunblotting and found cyanidin promoted the expression of anti-apoptotic proteins and inhibited the expression of pro-apoptosis protein Bak (Fig. 5D) and relative quantification was statistically significant (Fig. 5E and F).

Cyanidin suppresses cisplatin-induced ERK activation. ERK pathway plays an active role in cisplatin-induced apoptosis and caspase cascade signal activation to initiate apoptosis (20), but has not been confirmed in myocardial cells apoptosis model. To answer this doubt, H9c2 cells were incubated with different concentrations of cisplatin for $24 \mathrm{~h}$, and then detected by immunblotting (Fig. 6). The data showed that phosphorylation of ERK increased with the increasing of cisplatin concentration (Fig. 6A), and the ratio of pERK/ERK also gradually increased (Fig. 6C), which means cisplatin could activate the ERK $1 / 2$ pathway in a dose-dependent manner. To further understand the protective mechanism of cyanidin, H9c2 cells were pre-incubated with U0126, and then incubated with cisplatin or/and cyanidin to detect the expression of ERK and p-ERK. The results indicated that U0126 could significantly block the activation of ERK induced by cisplatin, and cyanidin had similar effects as U0126 (Fig. 6B and D). These data suggested that ERK signaling pathway might play an important role in cyanidin against apoptosis and protecting cardiomyocytes.

\section{Discussion}

Recently, cisplatin-induced cardiovascular toxicity has received increasing attention, its occurrence mechanism and effective measures still restricts the clinical use of cisplatin. In vitro and in vivo studies have demonstrated the critical role of oxidative stress and apoptosis in cardiomyocytes cytotoxicity (21-23). Also, studies have shown that cyanidin has a potential chemoprotective agent can attenuates cisplatin-induced nephrotoxicity and neurotoxicity $(16,17,24)$. Therefore, as a protective agent against oxidative stress and anti-apoptosis, cyanidin was used to study the mechanism of cytotoxicity of cisplatin. The results of MTT assay indicated that cyanidin significantly reduced cisplatin-induced $\mathrm{H} 9 \mathrm{c} 2$ cells damage.(Fig. 1). TUNEL assay was more intuitive to show the toxicity of cisplatin $(40 \mu \mathrm{M})$ for $\mathrm{H} 9 \mathrm{c} 2$ cells, while cyanidin $(80 \mu \mathrm{M})$ makes the rate of apoptosis decreased (Fig. 2), this protective effect has also demonstrated in models of oxidative stress-mediated amyloid beta neurotoxicity (25).

Cardiomyocytes with rich mitochondria is the power of energy metabolism. When cisplatin accumulates in the mitochondrial matrix, it causes a large amount of ROS production and mitochondrial dysfunction, which leading to increased mitochondrial permeability, pro-apoptotic factor release and initiate apoptosis. In the present study, we demonstrated that cisplatin combined with cyanidin treatment could reduce ROS production and mitochondrial membrane depolarization, and decrease cytochrome $c$ release and activation of caspase 3 . Cisplatin-induced apoptosis mainly involve the mitochondrial-mediated intrinsic pathway, death receptor-mediated pathway and endoplasmic reticulum pathway, the most closely related is intrinsic pathway $(10,26,27)$. When cisplatin enters the cell, the Bcl-2 family of pro-apoptotic proteins such as Bad and Bim are activated to induce Bax translocation to mitochondrial outer membrane and forming dimer with Bak, causing the release of apoptotic factors, Further activation of caspase 9, leading to apoptosis. In our study, caspase 9 and caspase 8 were tested and the results suggested that cisplatin induced apoptosis through the mitochondrial pathway, whereas cyanidin played a protective role via mitigating mitochondrial translocation of Bax, down-regulating pro-apoptotic protein, and up-regulating anti-apoptotic proteins (Figs. 3 and 4).

Depending on cell types and stimulis, the activation of ERK pathway plays a different role, including regulation of apoptosis, proliferation, autophagy and senescence (28). The regulation of ERK pathway has been confirmed in cisplatin-induced apoptosis of renal tubular epithelial cells, by regulating the translocation of Bax, the expression of pro-apoptotic protein and anti-apoptotic protein, further regulating the apoptosis through mitochondrial pathway $(16,29)$. Moreover, further evidence suggested that U0126 attenuated caspase-3-mediated apoptosis through inhibition of ERK pathway in the cisplatin-induced acute kidney injury model (30). Consistent with this conclusion, our study clarified the relationship between ERK pathway, Bax translocation, mitochondrial pathway and apoptosis after further confirmation of ERK pathway inhibitor U0126, and this may be the mechanism by which cyanidin exert its anti-apoptotic effects (Figs. 5, 6).

In conclusion, our study demonstrated that cyanidin reduced the cardiotoxicity induced by cisplatin via inhibiting the ROS-mediated apoptosis, and mitochondrial pathway and ERK signaling pathway may be the mechanism of its protective effect.

\section{Acknowledgements}

We thank Basic Medical Research Center of Nanjing Medical University for her excellent technical assistance and Dr. Zhou for careful reading and helpful criticism of the manuscript.

\section{References}

1. Dasari S and Tchounwou PB: Cisplatin in cancer therapy: Molecular mechanisms of action. Eur J Pharmacol 740: 364-378, 2014.

2. Schlumbrecht MP and Hehr K: Cisplatin-induced bradycardia and the importance of the QT interval. J Oncol Pharm Pract 21: 157-160, 2015.

3. Raja W, Mir MH, Dar I, Banday MA and Ahmad I: Cisplatin induced paroxysmal supraventricular tachycardia. Indian J Med Paediatr Oncol 34: 330-332, 2013.

4. Khan S, Chen CL, Brady MS, Parameswaran R, Moore R, Hassoun $\mathrm{H}$ and Carvajal RD: Unstable angina associated with cisplatin and carboplatin in a patient with advanced melanoma. J Clin Oncol 30: e163-e164, 2012. 
5. Ma H, Jones KR, Guo R, Xu P, Shen Y and Ren J: Cisplatin compromises myocardial contractile function and mitochondrial ultrastructure: Role of endoplasmic reticulum stress. Clin Exp Pharmacol Physiol 37: 460-465, 2010.

6. Czaykowski PM, Moore MJ and Tannock IF: High risk of vascular events in patients with urothelial transitional cell carcinoma treated with cisplatin based chemotherapy. J Urol 160: 2021-2024, 1998.

7. Saleh RM, Awadin WF, El-Shafei RA, Elseady YY, Wehaish FE and Elshal MF: Cardioprotective role of tadalafil against cisplatin-induced cardiovascular damage in rats. Eur J Pharmacol 765: 574-581, 2015.

8. Chirino YI and Pedraza-Chaverri J: Role of oxidative and nitrosative stress in cisplatin-induced nephrotoxicity. Exp Toxicol Pathol 61: 223-242, 2009.

9. Zhang W, Zhao L, Liu J, Du J, Wang Z, Ruan C and Dai K Cisplatin induces platelet apoptosis through the ERK signaling pathway. Thromb Res 130: 81-91, 2012.

10. Park MS, De Leon M and Devarajan P: Cisplatin induces apoptosis in LLC-PK1 cells via activation of mitochondrial pathways. J Am Soc Nephrol 13: 858-865, 2002.

11. Sheridan C, Brumatti G, Elgendy M, Brunet M and Martin SJ An ERK-dependent pathway to Noxa expression regulates apoptosis by platinum-based chemotherapeutic drugs. Oncogene 29: 6428-6441, 2010.

12. Galvano F, La Fauci L, Vitaglione P, Fogliano V, Vanella L and Felgines C: Bioavailability, antioxidant and biological properties of the natural free-radical scavengers cyanidin and related glycosides. Ann Ist Super Sanita 43: 382-393, 2007.

13. Cvorovic J, Tramer F, Granzotto M, Candussio L, Decorti G and Passamonti S: Oxidative stress-based cytotoxicity of delphinidin and cyanidin in colon cancer cells. Arch Biochem Biophys 501: 151-157, 2010.

14. Shih PH, Yeh CT and Yen GC: Anthocyanins induce the activation of phase II enzymes through the antioxidant response element pathway against oxidative stress-induced apoptosis. J Agric Food Chem 55: 9427-9435, 2007.

15. Park SY, Lee YK, Lee WS, Park OJ and Kim YM: The involvement of AMPK/GSK3-beta signals in the control of metastasis and proliferation in hepato-carcinoma cells treated with anthocyanins extracted from Korea wild berry Meoru. BMC Complement Altern Med 14: 109, 2014.

16. Gao S, Chen T, Choi MY, Liang Y, Xue J and Wong YS: Cyanidin reverses cisplatin-induced apoptosis in $\mathrm{HK}-2$ proximal tubular cells through inhibition of ROS-mediated DNA damage and modulation of the ERK and AKT pathways. Cancer Lett 333: 36-46, 2013.

17. Li DW, Sun JY, Wang K, Zhang S, Hou YJ, Yang MF, Fu XY, Zhang ZY, Mao LL, Yuan $\mathrm{H}$, et al: Attenuation of cisplatin-induced neurotoxicity by cyanidin, a natural inhibitor of ROS-mediated apoptosis in PC12 cells. Cell Mol Neurobiol 35: 995-1001, 2015.
18. Bottinger L, Guiard B, Oeljeklaus S, Kulawiak B, Zufall N, Wiedemann N, Warscheid B, van der Laan M and Becker T: A complex of Cox4 and mitochondrial Hsp70 plays an important role in the assembly of the cytochrome c oxidase. Mol Biol Cell 24: 2609-2619, 2013.

19. Camano S, Lazaro A, Moreno-Gordaliza E, Torres AM, de Lucas C, Humanes B, Lazaro JA, Milagros Gomez-Gomez M, Bosca L and Tejedor A: Cilastatin attenuates cisplatin-induced proximal tubular cell damage. J Pharmacol Exp Ther 334: 419-429, 2010.

20. Wang X, Martindale JL and Holbrook NJ: Requirement for ERK activation in cisplatin-induced apoptosis. J Biol Chem 275: 39435-39443, 2000.

21. Dugbartey GJ, Peppone LJ and de Graaf IA: An integrative view of cisplatin-induced renal and cardiac toxicities: Molecular mechanisms, current treatment challenges and potential protective measures. Toxicology 371: 58-66, 2016.

22. El-Sawalhi MM and Ahmed LA: Exploring the protective role of apocynin, a specific NADPH oxidase inhibitor, in cisplatin-induced cardiotoxicity in rats. Chem Biol Interact 207: 58-66, 2014

23. Al-Majed AA, Sayed-Ahmed MM, Al-Yahya AA, Aleisa AM, Al-Rejaie SS and Al-Shabanah OA: Propionyl-L-carnitine prevents the progression of cisplatin-induced cardiomyopathy in a carnitine-depleted rat model. Pharmacol Res 53: 278-286, 2006.

24. Ademiluyi AO, Oboh G, Agbebi OJ and Akinyemi AJ: Anthocyanin-rich red dye of hibiscus sabdariffa Calyx modulates cisplatin-induced nephrotoxicity and Oxidative stress in rats. Int J Biomed Sci 9: 243-248, 2013.

25. Wang Y,Fu XT, Li DW, Wang K, Wang XZ, Li Y, Sun BL, Yang XY, Zheng ZC and Cho NC: Cyanidin suppresses amyloid beta-induced neurotoxicity by inhibiting reactive oxygen species-mediated DNA damage and apoptosis in PC12 cells. Neural Regen Res 11: 795-800, 2016.

26. Jiang M, Wang CY, Huang S, Yang T and Dong Z: Cisplatin-induced apoptosis in p53-deficient renal cells via the intrinsic mitochondrial pathway. Am J Physiol Renal Physiol 296: F983-F993, 2009.

27. El-Awady el SE, Moustafa YM, Abo-Elmatty DM and Radwan A: Cisplatin-induced cardiotoxicity: Mechanisms and cardioprotective strategies. Eur J Pharmacol 650: 335-341, 2011.

28. Cagnol S and Chambard JC: ERK and cell death: Mechanisms of ERK-induced cell death-apoptosis, autophagy and senescence. FEBS J 277: 2-21, 2010.

29. Potočnjak I and Domitrović R: Carvacrol attenuates acute kidney injury induced by cisplatin through suppression of ERK and PI3K/Akt activation. Food Chem Toxicol 98: 251-261, 2016.

30. Jo SK, Cho WY, Sung SA, Kim HK and Won NH: MEK inhibitor, U0126, attenuates cisplatin-induced renal injury by decreasing inflammation and apoptosis. Kidney Int 67: 458-466, 2005.

This work is licensed under a Creative Commons Attribution-NonCommercial-NoDerivatives 4.0 International (CC BY-NC-ND 4.0) License. 